\title{
Evaluation and Optimization of Underwater Image Restoration Algorithms
}

\author{
Alan Le Boudec*, Artur Mkrtchyan*, Barbara Džaja ${ }^{\ddagger}$, Vincent Rodin* and Hai Nam Tran* \\ *University of Brest, Lab-STICC, CNRS, UMR 6285 \\ Email: le-boudec.alan@etudiant.univ-brest.fr, mkrtchyan.artur@etudiant.univ-brest.fr \\ rodin.vincent@univ-brest.fr, hai-nam.tran@univ-brest.fr \\ †University of Split, University Department of Professional Studies \\ Email: Barbara.Dzaja@oss.unist.hr
}

\begin{abstract}
On-board restoration of underwater images on embedded platforms such as marine drones is faced with many obstacles including image quality and real-time constraints. Confrontations met on the way vary from views to solution methods depending on the goals, such as underwater vehicle realtime control and positioning or underwater object recognition. In this research, five algorithms for underwater image restoration were studied and evaluated. In order to evaluate the quality of the performing algorithms nine evaluation criteria were used. Split into two types, the no reference metrics assesses only the quality of the image results, while the full reference criterion uses a reference image to estimate it. The calculation of these criteria allows each algorithm to be compared. Furthermore, the possibility to optimise algorithms in order to make them applicable to meet real-time requirements on embedded platforms was investigated.
\end{abstract}

\section{INTRODUCTION}

The aquatic domain is still insufficiently researched today. More than $80 \%$ of the very deep areas remain unexplored, and the environmental conditions complicate underwater research [12]. At depth of only 150 meters, 99\% of the sunlight is being absorbed [4]. From the perspective of underwater image processing, the concept of distance is different. Apart from light reflections a certain part of the color spectrum is absorbed. Further, rocking sea usually makes the image unreadable and unusable.

Nowadays, many programs can improve the quality of underwater images. Most of the research in the depths of the oceans is conducted with underwater vehicles. Those vehicles use cameras for auto positioning. However, to make the best usage of the images, they should be processed immediately. Thus, there is a real time constraint, as the speed of underwater data transmission is limited, and on-board processing is required. To enable real-time processing, all algorithms built into the underwater vehicle should be applicable in this context. In addition, quality results are essential for effective image processing, as recognition or object detection.

To address this problem, Our objective in this paper was to improve the quality of underwater images by applying the algorithms and afterwards to evaluate their real-time performance. In order to achieve that, approach used was to study five different algorithms and evaluated with nine evaluation criteria. Following that, the possibility of real time application was investigated, and the algorithms were optimized when it was possible.

The rest of the article is organised as follows. Section II presents the background and related work. Section III describes the approach presented in this paper for evaluating underwater image restoration algorithms, and section IV shows the obtained results. Section V concludes the article and discusses future work.

\section{BACKGROUND \& RELATED-WORK}

Extensive research has been done to compare underwater image restoration algorithms with various criteria. Hou et al. [7] presented eight criteria to evaluate their proposed algorithm and compare it to the existing ones. However, the solution is subjective and does not differentiate which one is the best. The approach used in this work is to objectively compare existing solutions, and also to be able to evaluate their realtime performances.

\section{A. Algorithms}

For the case study five algorithms were chosen: Underwater hazelines [3], Local color mapping and color transfer [13], Fusion enhancing [2], Backscatter removing [19], and Automatic red-channel underwater image restoration [5]. These algorithms use different means to enhance an underwater image.

1) Underwater Hazelines: The main idea given by Berman et al. [3] permeated through this algorithm is that in each color channel $c \in R, G, B$ the image at each pixel is composed of two components, attenuated signal and veiling light, which can be seen in Equation 1:

$$
I_{c}(\boldsymbol{x})=t_{c}(\boldsymbol{x}) J_{c}(\boldsymbol{x})+\left(1-t_{c}(\boldsymbol{x})\right) \cdot A_{c}
$$

Where bold denotes vectors, $\boldsymbol{x}$ is the pixel coordinate, $I_{c}$ is the acquired image value in color channel $c, t_{c}$ is the transmission of that color channel, and $J_{c}$ is the object radiance that needs to be restored. The global veiling-light component $A_{c}$ is the scene value in areas with no objects $\left(t_{c}=0, \forall c \in\{R, G, B\}\right)$. 
2) Local color mapping and color transfer: Protasiuk et al. [13] describe an approach which uses two methods together. The algorithm compares each pixel with a set of pixels whose underwater colour is already known. It computes and matches the co-variance of the input image and of a reference image. The both criteria are found in their objective function 2 :

$$
\begin{aligned}
& \min _{\boldsymbol{A}, \boldsymbol{b}} f(\boldsymbol{A}, \boldsymbol{b}) \stackrel{\text { def }}{=} \underbrace{\left\|\boldsymbol{A} \boldsymbol{X}-\boldsymbol{Y}+\boldsymbol{b} \mathbf{1}_{\mathbf{3}}^{\boldsymbol{T}}\right\|_{F}^{2}}_{\text {Local Color Mapping }}+ \\
& \frac{\lambda_{1}}{2} \underbrace{\left\|\boldsymbol{A} \boldsymbol{C}_{\boldsymbol{i}} \boldsymbol{A}^{\boldsymbol{T}}-\boldsymbol{C}_{\boldsymbol{r}}\right\|_{F}^{2}}_{\text {Global Color Transfer }}+\frac{\lambda_{2}}{2}\left(\|\boldsymbol{A}\|_{F}^{2}+\|\boldsymbol{b}\|_{2}^{2}\right)
\end{aligned}
$$

A third term has an aim to find the parameters $\mathrm{A}$ and $\mathrm{b}$ for which the function is minimal. They used a third term to scale the red color channel, furthermore to scale the function they used lambda parameter.

3) Fusion enhancing: Ancuti et al. [2] propose an alternative single image solution based on the principles of multiscale fusion. It aims at a simple and fast approach, capable of increasing the visibility of a wide variation of underwater videos and images. The framework mixes specific inputs and carefully chooses weights to overcome the limitations of such environments. This is generally true for underwater scenes properly lit by natural light.The enhancement strategy consists of three main steps: input assignment (derivation of inputs from the original underwater image), definition of weight measures and multi-scale fusion of inputs and weight measures. In Equation 3 the enhanced image version $R(x, y)$ is obtained by fusing the defined inputs with the weight measures at every pixel location $(x, y)$ :

$$
R(x, y)=\sum_{k=1}^{K} \bar{W}^{k}(x, y) I^{k}(x, y)
$$

4) Backscatter removing: Zhang and Chau [19] proposed to remove the baskscatter phenomena on the image. To accomplish that, the authors use the technology of fusion. They separate the input image into a reflectance and illuminance image. After that, the color and haze on the two images are corrected. In this way they applied gaussian and laplacian pyramid based on multi scale fusion. The general procedure diagram of backscatter removing algorithm is shown in Figure 1.

5) Automatic red-channel underwater image restoration: Galdran et al. [5] explain that the sea has the properties to distort light and absorb specific wavelengths. They proposed an approach to restore an underwater image by finding missing colours. The red channel method is based on the work of dark colour channel which uses it for degrading an image with haze gained from atmospheric model.

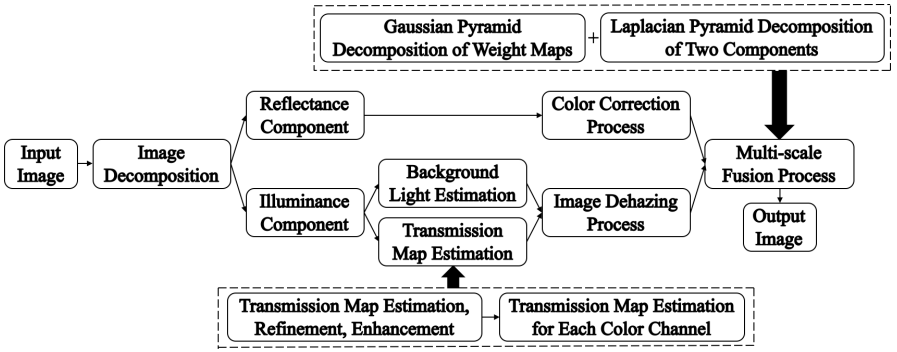

Fig. 1: The general procedures of objects visibility enhancement process

\section{B. Evaluation criteria}

In order to evaluate the quality of the image provided by each algorithm, it is necessary to determine the evaluation criteria. In this work, the evaluation criteria presented in [7] for the quality assessment was used. They are Blind/Referenceless Image Spatial Quality Evaluator (BRISQUE) [9], Naturalness Image Quality Evaluator (NIQE) [10], Underwater Image Quality Measures (UIQM) [11], Peak Signal to Noise Ratio (PSNR), Visual Information Fidelity (VIF) [15], Information Fidelity Criterion (IFC) [16] and Structural Similarity (SSIM) [18] based on [7]. Then two other criteria are added: the Second Norm or Euclidean distance [8] and Mean Square Error (MSE) [14]. The metrics are separated into two types, the full reference and no reference.

1) Full reference criteria: Full reference criterion compares two images using mathematical calculations. The first parameter is the image obtained by the algorithm that is being evaluated and the second parameter is the reference image. In this case the reference image is the original.

For the full reference criterion the most important is MSE which denotes the average difference of the pixels all over the image. A higher value represents a significant difference between the images, i.e. the image gained with the algorithm is far from the original (reference) image. PSNR is a measure of image distortion, it measures the ability to remove noise from an image. Higher value of PSNR means better image quality. SSIM is normally employed to measure the recovered information of light, contrast and structure. This is related to Universal Quality Image (UQI) [17] criteria, and like previous criterion, it is better if the value is high. VIF is an image quality evaluation index proposed by combining natural image statistical model, image distortion model and human visual system mode. IFC is used for image quality assessment using natural scene statistics and the notion of image information extracted by the human visual system. High VIF and IFC values mean good results.

2) No reference criteria: No reference metrics evaluates the quality of a single image based on mathematical calculations. Hence, the only parameter is the image from the algorithm that needs to be evaluated.

The no reference criteria have fewer terms. BRISQUE measures the image quality by using locally normalized light coefficients which were used to calculate the image features. 

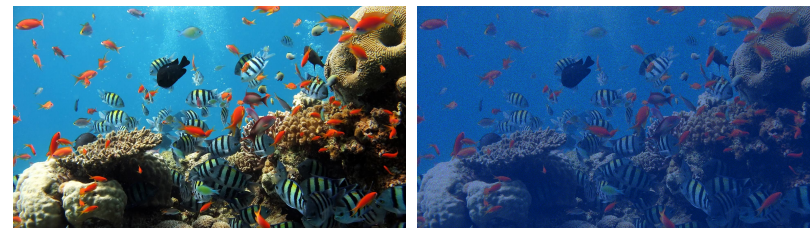

Fig. 2: degradation of an image [1]

NIQE is completely blind image quality analyzer that only makes use of measurable deviations from statistical regularities observed in natural images. For NIQE and BRISQUE a small value indicates better result.

UIQM is based on three underwater image attribute measures: the underwater image colourfulness measure (UICM), the underwater image sharpeness measure (UISM), and the underwater image contrast measure (UIConM). This third measure is explained by Panetta et al. in [11]. Higher the value better the result.

\section{APPROACH}

\section{A. Benchmark}

For testing the algorithms 20 underwater images were chosen. The size of these images varies on purpose in order to perceive how algorithms evolve. Each one was chosen for its good image quality, so the idea behind was to anticipate the results gained with the algorithms. Good quality images were degraded by image degradation model that consisted of added noise and a blue filter which acted as the colour of the water. These degraded images formed an image base for algorithm. The results obtained by the algorithms are compared to the original images. Example of one degraded image can be seen in Figure 2.

However, testing revealed some limitations of the algorithms. Underwater Hazelines algorithm did not work for two of the images, and the rest of the algorithms did not work for other three. Very small images do not work properly with all the algorithms. Hence, a total of 15 images were used as image testing base.

Afterwards to test correctly, it had to be checked whether some of the algorithms used other input parameters than just image that had to be improved. This is the case for Local Color Mapping and Color Transfer [13] which uses a reference image. Hence, the best reference image needs to be chosen in order to get the best result. The proposed way of how to do this presented in this paper is to compute the criteria values (PSNR and Euclidean distance) on all reference images given by the data set of [13] and choose the best image that has the best result.

\section{B. Criteria evaluation}

Different methods to improve underwater image are compared with each other regarding selected criteria. These criteria allow to evaluate the quality of an image either with a reference image or just the image to be evaluated. To compare them, each criterion is calculated, some of them by Matlab function or by equations given in research articles. Evaluation of the best solution is done in the form of a score. It means that an algorithm has 1 point if it's the best on one criteria on one image. Whichever has the most points is designated as the one with the highest quality.

\section{Optimization}

Above-mentioned algorithms are focused on the image processing aspect and the quality of image restoration which implies the neglect of the processing speed and a possible application for video stream processing. In order to better adapt the algorithms to the video stream processing criteria, several improvements were applied:

- The first tendency was the improvement of the source code.

- Cleaning of unnecessary functions and parameters to make it as compact as possible.

- Code adaptation to extract the existing GUI (e.g. Local color mapping).

- The second tendency was to improve the speed of image processing because it directly impacted on the speed of video stream processing.

- Most of the algorithms use the LAB format during image processing. That implies transforming from RGB to $L A B$ at the beginning of the processing for each image and transforming back from $\mathrm{LAB}$ to RGB at the end of the algorithm. The processing time for these two transformations is not negligible in comparison with a total processing time of an image. Therefore, to improve this part of the processing, Colorspace Transformation library [6] (C language) was implemented in Matlab code (the results are presented in Table II).

- Finally, the last tendency was the adaptation of the best suited algorithm to video processing (in terms of quality, Fusion [2] algorithm has been chosen).

- Firstly, a function that will apply the algorithm in a loop on each frame of the video stream had to be created.

- Secondly, the parallelism of processing needed to be implemented to improve the processing speed.

\section{EVALUATION}

This section explains two experiments that were performed. The first experiment was to measure the quality and make comparisons between the algorithms. In the second experiment, the execution time was calculated in order to optimise for a possible real time application.

\section{A. Experiment 1}

The human visual system is not perfect. If one takes a look at Figure 3 it is obviously complicated to objectively choose the best result. The first experiment allows user to evaluate the quality of images obtained by each algorithm. This assessment is made by calculating the values of the criteria for each 


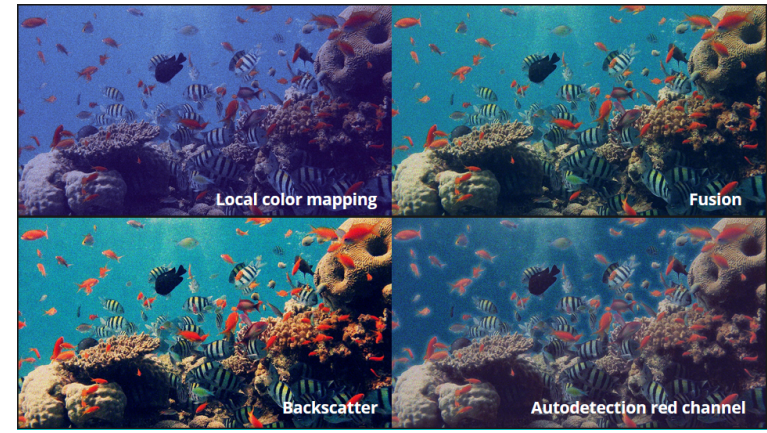

Fig. 3: Results images

algorithm.

The legend used for the graphs is described in Figure 4.

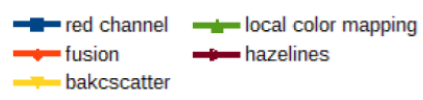

Fig. 4: Legend

Figure 5 shows no reference results. For BRISQUE and NIQE Automatic Red-channel Underwater Image Restoration seems to be the best. However, for UIQM Local Color Mapping and Color Transfer has good results. Knowing there are only 3 no reference criteria, The results present Automatic Redchannel Underwater Image Restoration as the best solution.

Full reference testing in Figure 6 shows that with some criteria it is difficult to see which algorithm is the best, like for IFC or NORM. However, for PSNR Fusion is the best one, and in the case of MSE it is always Fusion that has the lowest value, and for SSIM criteria the highest value is Fusion too. With exception of two criteria IFC and NORM, on average, it is Fusion that has the best results.

Figure 7 presents all results in different way. Ordinate value is the number of time which the algorithm performed the best. This means that when an algorithm has the best result it gets 1 point for each processed image.

On this graphic, one algorithms stands out. Fusion gives 4 times the best result, with respect to PSNR, SSIM, IFC and MSE. However for BRISQUE, NIQE and NORM metrics Automatic Red-channel Underwater Image Restoration shows better quality. The rest of the algorithms performed poorly. All in all, Fusion is the best relying on 4 criteria. In average, it is the one with the best image quality.

\section{B. Experiment 2}

In order to apply algorithm in real time context time constraints had to be met. The run-time measurement must be of a low enough value to be used. In that respect, these values are computed for all images from the benchmark as shown in Table I.

The data was obtained with the Matlab software, having setup the following working environment: OS Ubuntu 18.04, Intel(R) Core(TM) i7-4790 CPU @ 3.60GHz, 16 GB of RAM.
TABLE I: 5 algorithms execution times in seconds for 15 image restorations

\begin{tabular}{|l|l|l|l|l|l|}
\hline Images & $\begin{array}{c}\text { Autodection } \\
\text { red color channel }\end{array}$ & $\begin{array}{c}\text { Fusion } \\
\text { enhancing }\end{array}$ & $\begin{array}{c}\text { Backscatter } \\
\text { remove }\end{array}$ & $\begin{array}{c}\text { Local color } \\
\text { mapping and } \\
\text { color transfer }\end{array}$ & Hazclines \\
\hline 1 & 2.38 & 11.07 & 5.3 & 3.58 & 223.19 \\
\hline 2 & 0.81 & 2.25 & 2.51 & 1.73 & 125.14 \\
\hline 3 & 7.25 & 15.63 & 16.93 & 12.45 & 737.86 \\
\hline 4 & 1.01 & 2.85 & 2.94 & 2.31 & 153.89 \\
\hline 5 & 0.79 & 2.18 & 2.39 & 1.86 & 116.46 \\
\hline 6 & 0.08 & 0.33 & 0.27 & 0.18 & 12.53 \\
\hline 7 & 0.31 & 0.92 & 0.86 & 0.62 & 43.81 \\
\hline 8 & 0.32 & 0.94 & 0.93 & 0.66 & 46.92 \\
\hline 9 & 0.96 & 2.42 & 2.69 & 1.89 & 134.86 \\
\hline 10 & 0.09 & 0.41 & 0.31 & 0.19 & 13.86 \\
\hline 11 & 0.11 & 0.43 & 0.33 & 0.27 & 17.04 \\
\hline 12 & 0.25 & 0.86 & 0.77 & 0.54 & 35.19 \\
\hline 13 & 0.69 & 1.78 & 2.08 & 1.41 & 98.57 \\
\hline 14 & 0.94 & 2.38 & 2.93 & 1.98 & 138.49 \\
\hline 15 & 0.07 & 0.34 & 0.23 & 0.17 & 11.16 \\
\hline
\end{tabular}

All times are expressed in seconds. The figures show that the solution with the shortest execution time is Automatic Redchannel Underwater Image Restoration. This method has a good evaluation on the quality of its' images, but it is not the best. Furthermore, its' values are ideally close to the time required for a possible real time application, hence it is taken as the baseline* ${ }^{*}$. Hazeline takes an extremely long time, which is almost 20 times longer than others. Even with optimization it would be difficult to achieve an acceptable result with Hazeline.

Furthermore, the baseline* is used to calculate the percentage of loss for the other methods. Figure 8 presents comparison of Fusion, Local Color Mapping and Color Transfer and Backscatter remove without Hazeline. $Y$ axis presents the percentage of loss compared to baseline* ${ }^{*}$ and $X$ axis shows the image number. Fusion which has the best image quality rating has also the highest percentage of execution time, in average $231 \%$. Backscatter has a mean percentage of $196 \%$ and $111 \%$ for Local Color Mapping and Color Transfer. This algorithm for its quality results has been optimized to be potentially applicable for a real time target.

The optimization of Fusion performed in this research is summarized in Table II. It shows gain of $37 \%$ in average. For instance, the first image takes 11,07 seconds and when it is optimized, it takes only 6,89 seconds, hence 4,18 seconds are gained (or 38\%).

Another way that benefits of optimization become visible is to review the resulting Figure II with new times gained with optimized algorithm, and it is presented in Figure 9. The curve of optimized Fusion algorithm is below the curves of other two algorithms. This expresses an improvement between 

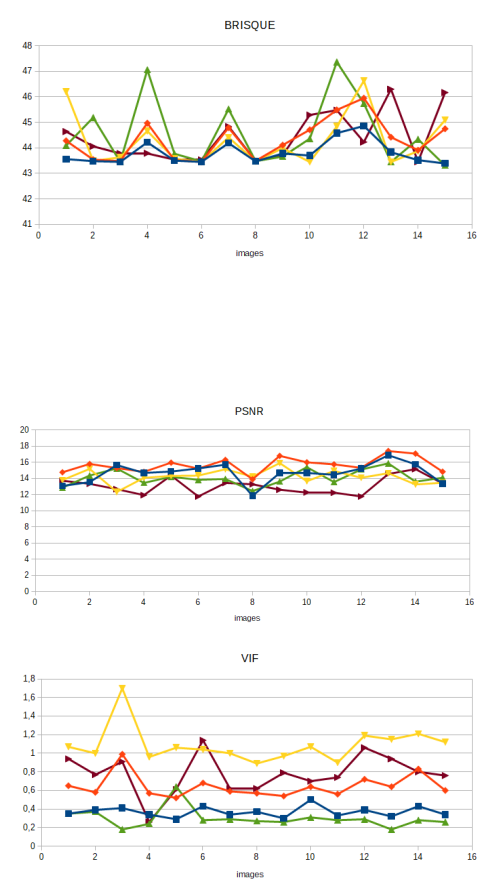

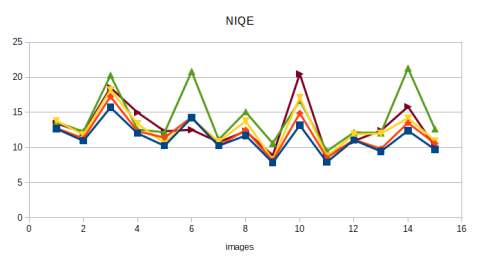

Fig. 5: No Reference Criteria Results
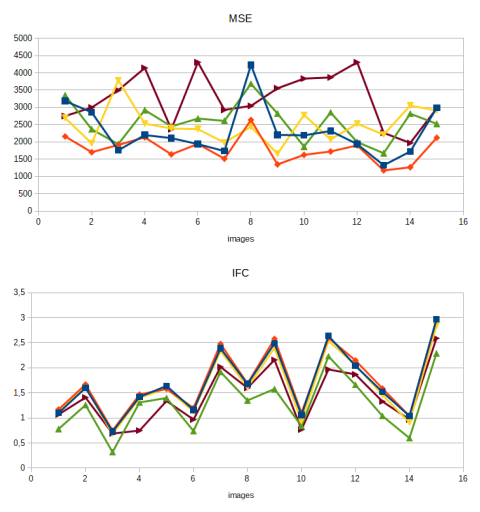

Fig. 6: Full Reference Criteria Results
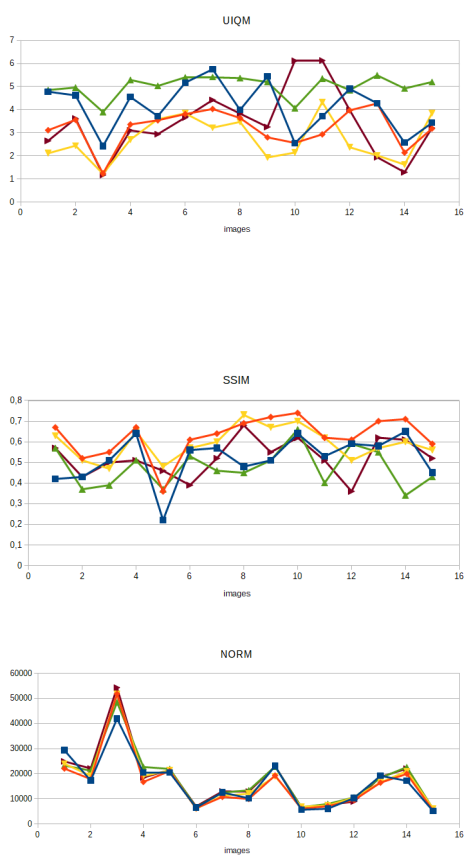

TABLE II: Fusion algorithm execution time for 15 images restoration

\begin{tabular}{|l|l|l|l|}
\hline Images & Original & Optimize & Gain \% \\
\hline 1 & 11.07 & 6.89 & 38 \\
\hline 2 & 2.25 & 1.71 & 24 \\
\hline 3 & 15.63 & 10.13 & 35 \\
\hline 4 & 2.85 & 1.85 & 35 \\
\hline 5 & 2.18 & 1.51 & 31 \\
\hline 6 & 0.33 & 0.19 & 42 \\
\hline 7 & 0.92 & 0.57 & 38 \\
\hline 8 & 0.94 & 0.63 & 33 \\
\hline 9 & 2.42 & 1.7 & 30 \\
\hline 10 & 0.41 & 0.21 & 49 \\
\hline 11 & 0.43 & 0.25 & 42 \\
\hline 12 & 0.86 & 0.48 & 44 \\
\hline 13 & 1.78 & 1.35 & 24 \\
\hline 14 & 2.38 & 1.74 & 27 \\
\hline 15 & 0.34 & 0.18 & 47 \\
\hline
\end{tabular}

Figure 8 and Figure 9. In the first graph, the curve is clearly above all other curves, but with proposed optimization it passes completely below.

Finally, all these results consist of image processing. Optimizing the video processing by parallelizing the frame processing can result with more efficient results. For a video file sized 1920x1080 and with duration of 17 seconds (approximately 30 frames per second and 530 frames in total) the

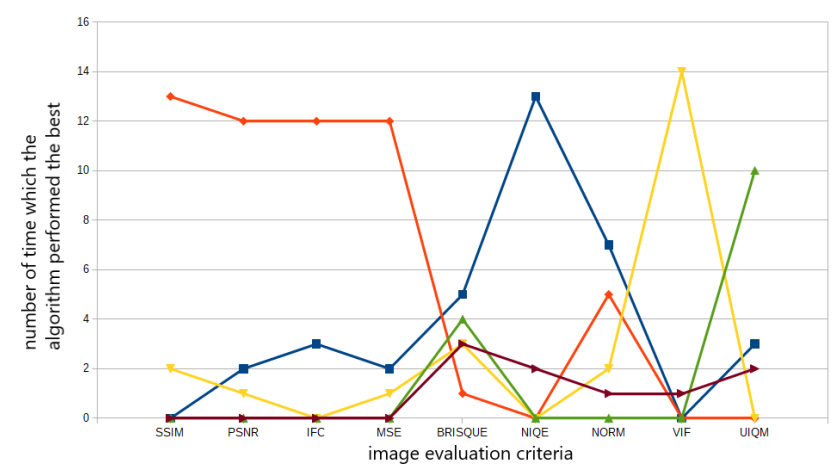

Fig. 7: Graphics results

results are as follows. In sequential version 1 frame processing in average takes 2.3 seconds. In parallel version 1 frame processing in average take 1.21 seconds (Gain of 47,4\%).

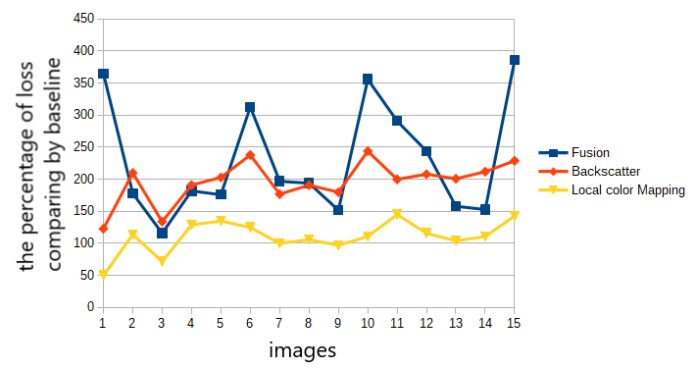

Fig. 8: Percentage of loss 


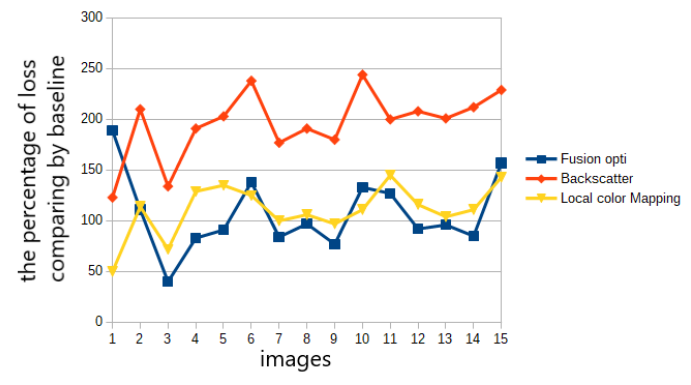

Fig. 9: Percentage of loss with Fusion Optimize

\section{Evaluation}

The evaluation of algorithms with these different criteria reveals the following. Fusion and Automatic Red-channel Underwater Image Restoration algorithms have the best results. On the average of all criteria the Fusion algorithm outperforms all others. Judging on the full reference criteria Fusion is the best and in the field of no reference Automatic Redchannel Underwater Image Restoration gives the best result. Experiments performed in this research conclude that two algorithms stand out. The other part of the evaluation showed that Local color mapping and color transfer and Underwater Hazelines are the worst in terms of quality.

In large, the research showed that there are two algorithms with good results. Furthermore, when their execution time is compared in order to see if it is possible to apply them in a real time context, it stands out that Automatic Red-channel Underwater Image Restoration is much faster than Fusion. On all the images the algorithm takes about 1 second in average to process the image while Fusion takes about 3 seconds. Hence, Automatic Red-channel Underwater Image Restoration can be applied to real time images under certain conditions, i.e. on images of average quality and size it performs effectively and adequately by virtue of its power to combine processing quality and efficiency.

However, for Fusion it had to be optimized to achieve this efficiency ( 3 seconds for one image is too long). Knowing that in video processing there are about 30 frames per second, this would mean 1.5 minutes for 1 second of video.

Image processing optimisation gives very interesting results with gain of $37 \%$ in average. Video processing optimisation using parallelism additionally improves the processing speed with gain of $47,4 \%$ in average. However, it is always too long for the objective goal and still not applicable for dealing with real time constraints.

\section{CONCLUSION}

This paper demonstrates how to evaluate the quality of images gained with image processing algorithms and if it was possible to apply them to real time targets. The comparison by evaluation criteria highlighted Fusion with the best results and Automatic Red-channel Underwater Image Restoration which has a good runtime, unlike Fusion. For this reason Fusion was optimized. In the basics of image processing optimization of the algorithm manifested with significant time saving of around $37 \%$. Further, in video processing optimization processing time was improved by $47,4 \%$.

The advantage of optimizing Fusion algorithm was undoubtedly in meeting the time constraints. This way showed how possible it was to run this algorithm on real time systems. By applying the proposed approach the perfect algorithm was singled out by its' quality and capability to increase its performance in terms of time and hence acquired satisfactory results.

The results of the execution times were calculated on computers. Thereafter, the next step should be to test the algorithms directly on real time targets. This way the quality results and time execution could be evaluated in real environment.

\section{REFERENCES}

[1] Great Barrier Reef fish. https://www.newton.com.tw/wiki/.

[2] Cosmin Ancuti, Codruta Orniana Ancuti, Tom Haber, and Philippe Bekaert. Enhancing underwater images and videos by fusion. In 2012 IEEE conference on computer vision and pattern recognition, pages 8188. IEEE, 2012.

[3] Dana Berman, Tali Treibitz, and Shai Avidan. Diving into haze-lines: Color restoration of underwater images. In Proc. British Machine Vision Conference (BMVC), volume 1, 2017.

[4] Dive \& Discover. Underwater Research Vehicles,How Deep Can We Go? https://divediscover.whoi.edu/underwater-vehicles/how-deep-canwe-go/, 2021.

[5] Adrian Galdran, David Pardo, Artzai Picón, and Aitor Alvarez-Gila. Automatic red-channel underwater image restoration. Journal of Visual Communication and Image Representation, 26:132-145, 2015.

[6] Pascal Getreuer. Colorspace Transformations. version 1.4, 2021.

[7] Guojia Hou, Xin Zhao, Zhenkuan Pan, Huan Yang, Lu Tan, and Jingming Li. Benchmarking underwater image enhancement and restoration, and beyond. IEEE Access, 8:122078-122091, 2020.

[8] MD Malkauthekar. Analysis of euclidean distance and manhattan distance measure in face recognition. In Third International Conference on Computational Intelligence and Information Technology (CIIT 2013), pages 503-507. IET, 2013.

[9] Anish Mittal, Anush Krishna Moorthy, and Alan Conrad Bovik. Noreference image quality assessment in the spatial domain. IEEE Transactions on image processing, 21(12):4695-4708, 2012.

[10] Anish Mittal, Rajiv Soundararajan, and Alan C Bovik. Making a "completely blind" image quality analyzer. IEEE Signal processing letters, 20(3):209-212, 2012.

[11] Karen Panetta, Chen Gao, and Sos Agaian. Human-visual-systeminspired underwater image quality measures. IEEE Journal of Oceanic Engineering, 41(3):541-551, 2015.

[12] EMILY PETSKO. Why does so much of the ocean remain unexplored and unprotected? https://oceana.org/blog/why-does-so-muchocean-remain-unexplored-and-unprotected, 2020.

[13] Rafał Protasiuk, Adel Bibi, and Bernard Ghanem. Local color mapping combined with color transfer for underwater image enhancement. In 2019 IEEE Winter Conference on Applications of Computer Vision (WACV), pages 1433-1439. IEEE, 2019.

[14] Mark D Schluchter. Mean square error. Encyclopedia of Biostatistics, $5,2005$.

[15] Hamid R Sheikh and Alan $\mathrm{C}$ Bovik. Image information and visual quality. IEEE Transactions on image processing, 15(2):430-444, 2006.

[16] Hamid R Sheikh, Alan C Bovik, and Gustavo De Veciana. An information fidelity criterion for image quality assessment using natural scene statistics. IEEE Transactions on image processing, 14(12):21172128, 2005.

[17] Zhou Wang and Alan C Bovik. A universal image quality index. IEEE signal processing letters, 9(3):81-84, 2002.

[18] Zhou Wang, Alan C Bovik, Hamid R Sheikh, and Eero P Simoncelli. Image quality assessment: from error visibility to structural similarity. IEEE transactions on image processing, 13(4):600-612, 2004.

[19] Hao Zhang and LP Chau. Removing Backscatter to Enhance the Visibility of Underwater Object. PhD thesis, Master's Thesis, Nanyang Technological University, Singapore, 2016. 